\title{
Los servicios de genética médica en Venezuela
}

\author{
Carlos A. De La Torre-Hernandez, ${ }^{1}$ Yvonne Guedez, ${ }^{2}$ Lennie Pineda-Bernal, ${ }^{3}$ \\ Héctor A. Ojeda y y Yuliana A. Guevara-Guerra ${ }^{5}$
}

Forma de citar De La Torre-Hernandez CA, Guedez Y, Pineda-Bernal L, Ojeda HA, Guevara-Guerra YA. Los servicios de genética médica en Venezuela. Rev Panam Salud Publica. 2018;42:e78. https://doi.org/10.26633/ RPSP.2018.78

RESUMEN Objetivo. Caracterizar los servicios de genética médica de Venezuela con el fin de conocer la distribución de sus recursos, servicios, tecnologías y formación profesional.

Métodos. Se realizó una investigación descriptiva, de tipo documental, entre febrero y noviembre de 2016 de los servicios de genética, mediante la revisión de fuentes documentales primarias y el uso de una ficha de recolección de datos en las instituciones de investigación para información referente a disponibilidad de recursos humanos, servicios de atención y diagnóstico, así como formación profesional, y la base de datos de la Sociedad Venezolana de Genética Humana, que permitió identificar los recursos humanos en centros de genética. El criterio de inclusión fue instituciones con recursos humanos formados en genética.

Resultados. Los criterios fueron cumplidos por cuatro instituciones de investigación, siete universidades y cuatro hospitales, todos del sector público. En estas instituciones trabajan 124 profesionales, 56 son médicos y 68 se desempeñan en el área de laboratorio. Sesenta y dos por ciento de los profesionales pertenecen a las instituciones de investigación; estas cuentan con servicios de atención clínico, diagnóstico molecular, bioquímico y, con menos frecuencia, los análisis citogenéticos, prenatales y forenses. Cinco regiones del país tienen entre dos y cuatro médicos genetistas por millón de habitantes. El 96\% de los profesionales de laboratorio se localizan en dos regiones (Capital y Zuliana), cinco regiones carecen de ellos. Las instituciones de investigación han formado en genética el $40 \%$ de los recursos humanos actuales del país.

Conclusiones. Los servicios de genética presentan gran variabilidad de opciones diagnósticas, un acceso limitado y grandes aportes en formación profesional; se requieren políticas coordinadas que los integre y disminuya las brechas.

Palabras clave Genética médica; servicios genéticos; recursos humanos; Venezuela.

La genética, como disciplina científica, tiene sus inicios entre la sexta y octava décadas del siglo XIX, con el planteamiento

\footnotetext{
Ministerio del Poder Popular para la Salud, Caracas, Venezuela. Enviar correspondencia a Carlos A. De La Torre-Hernandez, dr.delatorre@gmail.com

2 Servicio Autónomo Instituto de Altos Estudios "Dr. Arnoldo Gabaldón", Aragua, Venezuela.

3 Instituto de Investigaciones Genéticas "Dr. Heber Villalobos Cabrera", Universidad del Zulia, Maracaibo, Venezuela.
}

de sus bases generales (1). La genética médica derivada de la genética humana es la ciencia de las variaciones biológicas

\footnotetext{
4 Laboratorio de Genética Humana, Instituto Venezolano de Investigaciones Científicas (IVIC), Miranda, Venezuela.

5 Unidad de Errores Innatos del Metabolismo,

Fundación Instituto de Estudios Avanzados (IDEA), Miranda, Venezuela.
}

humanas y su relación con la salud y la enfermedad. La genética clínica es la parte de la genética médica que relaciona la salud del individuo y sus familiares, se define como la práctica de la ciencia del diagnóstico, prevención y manejo de los desórdenes genéticos (2).

Los servicios de genética médica están constituidos por instituciones de salud dedicadas al diagnóstico, tratamiento,

Este es un artículo de acceso abierto distribuido bajo los términos de la licencia Creative Commons Attribution-NonCommercial-NoDerivs 3.0 IGO, que permite su uso, distribución y reeproducción en cualquier medio, siempre que el trabajo original se cite de la manera adecuada. No se permiten modificaciones a los artículos ni su uso comercial. Al reproducir un artículo no debe haber ningún indicio de que la OPS o el artículo avalan a una organización o un producto específico. El uso del logo de la OPS no está permitido. Esta leyenda debe conservarse, junto con la URL original del artículo. 
seguimiento y asesoramiento de personas y sus familiares, afectados o en riesgo de enfermedades genéticas $(3,4)$. Las instituciones de genética se ubican en regiones con dos a cinco millones de habitantes y tienen capacidad para atender de 1000 a 3000 consultas anuales. Estos servicios iniciaron siendo parte de la atención terciaria por su nivel de especialización (5). Los posteriores avances han generado un incremento de sus aplicaciones en la atención primaria (6).

Los servicios de genética se organizan con la integración de áreas clínicas y de laboratorio (4). En las áreas clínicas, se establece el contacto entre el usuario y los profesionales de salud; suelen estar atendidas por médicos especialistas en genética clínica o entrenados en genética, también conocidos como médicos genetistas; además de otros profesionales de salud con formación en la especialidad. En los laboratorios de diagnóstico se encuentran los profesionales de laboratorio, conocidos como genetistas de laboratorio, en su mayoría licenciados en biología, bioanálisis, bioquímica o genética. Los servicios se dividen en asistenciales-preventivos de base individual-familiar, enfocado en la atención directa al usuario; y programas de genética de base poblacional o salud pública genética (5).

En los últimos 50 años, la región de América Latina y el Caribe ha mejorado los indicadores de salud. El urbanismo, la modernización, los nuevos hábitos alimentarios y el estrés han generado una transición epidemiológica; esto ha incrementado la proporción de morbilidad y mortalidad por defectos congénitos, enfermedades genéticas y enfermedades crónicas asociado a predisposición genética (7). Los servicios de genética médica han tenido un desarrollo fragmentado y desigual, concentrados en zonas urbanas y ricas. Se calcula que el número de genetistas clínicos es cerca de uno por millón de habitantes (8).

En Venezuela, el Sistema Nacional de Salud está integrado por los sectores público y privado. El Sistema Público Nacional de Salud (SPNS) está basado en los principios de la Constitución Nacional de 1999, donde la salud es un derecho social fundamental que el Estado garantiza a partir de un sistema sanitario de servicios gratuitos, único, universal, descentralizado y participativo (9). Está conformado por más de 14000 centros de salud y tiene como ente rector el Ministerio del Poder Popular para la
Salud (MPPS). El SPNS se ejecuta mediante dos modelos: la red de atención tradicional y la red Barrio Adentro. La primera está gestionada por dos tipos de direcciones regionales de salud, centralizadas y descentralizadas, estructurada en tres niveles de atención, y organizada en redes de ambulatorios, hospitales y centros de mediana y alta complejidad. La segunda se organiza en Áreas de Salud Integral Comunitaria (ASIC). Ambos componentes se hallan hoy en día en proceso de fusión administrativa. Por otra parte, se cuenta con otros subsistemas de salud, conformados por el Instituto Venezolano de los Seguros Sociales (IVSS), el Instituto de Previsión y Asistencia Social del Ministerio de Educación (IPASME), el Instituto de Previsión Social de las Fuerzas Armadas (IPSFA), la Corporación Venezolana de Guayana (CVG) y Petróleos de Venezuela S.A. (PDVSA).

Los estudios sobre genética humana en Venezuela iniciaron en 1969 (10) en el Instituto Venezolano de Investigaciones Científicas (IVIC), específicamente en el Laboratorio de Genética Humana (LGH-IVIC). Le siguieron el Instituto de Investigaciones Genéticas "Dr. Heber Villalobos Cabrera" de la Facultad de Medicina de la Universidad del Zulia (IIG-LUZ) y la Fundación Instituto de Estudios Avanzados (IDEA) con una Unidad de Errores Innatos del Metabolismo (UDEIM-IDEA) fundados en 1973 (11) y 1979 (12), respectivamente. En 2009, se creó el Centro Nacional de Genética Médica de Venezuela "Dr. José Gregorio Hernández" (CNGMV), enfocado en la genética comunitaria, con la particularidad de contar con profesionales de origen cubano como parte de un convenio de cooperación (13). El recurso humano se distribuye en estas instituciones así como en unidades, laboratorios y departamentos de universidades públicas como la Universidad Central de Venezuela (UCV) y la Universidad Simón Bolívar (USB) en Caracas, la Universidad de los Andes (ULA) en Mérida, la Universidad Centroccidental Lisandro Alvarado (UCLA) en Barquisimeto, la Universidad de Carabobo (UC) núcleos Valencia y Aragua, la Universidad de Oriente (UDO) núcleo Ciudad Bolívar, además de la Universidad del Zulia (LUZ), en Maracaibo a donde pertenece el IIG-LUZ. Asimismo, existen recursos capacitados en hospitales públicos y médicos genetistas que ejercen en privado. La primera unidad de genética médica fue creada en 1971 en LUZ. En 1977 se funda la Sociedad Venezolana de Genética y cinco años después es reconocida la genética clínica como especialidad médica (14).

Las instituciones con servicios de genética se caracterizan por tener gran variabilidad estructural y funcional; la falta de mecanismos que garanticen su trabajo coordinado puede generar un aumento en las brechas de inequidad en el acceso y una ineficiencia en la utilización de recursos (15). En tal sentido, es indispensable conocer el funcionamiento de las instituciones, sus recursos y el aporte al desarrollo de la disciplina. En Venezuela, la inserción de estas instituciones en el SPNS garantiza su consolidación, de esta manera fortalece el nivel más elevado de atención en salud. El objetivo de este trabajo es caracterizar los servicios de genética médica de Venezuela con el propósito de conocer el acceso a los servicios, distribución de recursos humanos, servicios y tecnologías disponibles, así como el aporte en la formación de recursos con respecto a la región de América Latina y el Caribe.

\section{MATERIALES Y MÉTODOS}

Se realizó una investigación descriptiva y documental. La unidad de análisis estuvo constituida por todas las instituciones de investigación en genética y centros que brinden servicios de genética médica en Venezuela. Como criterio de inclusión se utilizó las instituciones con recursos humanos formados en genética. La técnica de recolección de datos fue la revisión de fuentes documentales primarias. Como instrumento se utilizó una ficha de recolección de datos, la cual estuvo conformada por cuatro preguntas con diferentes secciones cerradas; todas las secciones contaron con un acápite abierto de aclaración. Los indicadores definidos para determinar los recursos humanos según la institución fueron: número de médicos genetistas y número de genetistas de laboratorio, en ambos casos según el nivel de formación. Para conocer la disponibilidad de servicios de atención, se utilizaron consultas de genética médica, consultas médicas especializadas relacionadas y consultas profesionales relacionadas. En el caso de los servicios diagnóstico se consideraron: diagnóstico citogenético, diagnóstico molecular, genética forense, diagnóstico prenatal, 
diagnóstico prenatal no invasivo y errores congénitos del metabolismo mediante pruebas bioquímicas. Para determinar los recursos formados en genética según institución se usó el número de profesionales graduados según programa de formación. La recolección de datos se realizó entre el 1 febrero y el 30 de noviembre de 2016 en las cuatro instituciones de investigación en genética. La información de los centros de genética se obtuvo de la base de datos actualizada al 2016 de la Sociedad Venezolana de Genética Humana. El análisis de la disponibilidad de recursos incluyó las nueve regiones del territorio nacional: de los Andes, Capital, Central, Centro-Occidental, de Guayana, Insular, de los Llanos, Nor-Oriental y Zuliana $(16,17)$. Los resultados se analizaron bajo los parámetros de la estadística descriptiva para comprender el comportamiento de las variables.

\section{RESULTADOS}

En Venezuela, los recursos humanos formados en genética médica en los sectores públicos y privado suman 131 profesionales; perteneciendo al sector público 124 (95\%); de estos, 56 son médicos y 68 son profesionales de laboratorio. En las instituciones de investigación se localizan 77 profesionales, 26 (34\%) en el IIG-LUZ, 19 con maestría en ciencias (MSc por sus siglas en inglés) y 11 con título de doctor en ciencias médicas de LUZ; le sigue el CNGMV con 23 (30\%) profesionales, 19 son médicos con nivel de MSc. El LGH-IVIC cuenta con 16 (21\%) profesionales, 5 con posdoctorado, y la UDEIM-IDEA dispone de 12 (15\%) profesionales en su mayoría de laboratorio. El departamento de biología celular de la USB tiene el mayor número de profesionales de laboratorio, que represen$\tan 37 \%$ de los genetistas de laboratorio del país. Aproximadamente la mitad de los servicios de genética médica ofrecidos en el resto de universidades $-\mathrm{UCV}$, ULA, UCLA, UC y UDO- y en cuatro hospitales ubicados en tres estados -Distrito Capital, Trujillo y Falcón- se efectúan en unidades de genética que basan sus recursos en personal médico con nivel de MSc (cuadro 1). Los siete (5\%) profesionales que ejercen en el sector privado son médicos genetistas y lo hacen en su mayoría en el Distrito Capital.
Los servicios de atención en genética médica públicos se concentran en las instituciones de investigación, tres de estas tienen al menos una consulta de genética médica (CGM), la UDEIM-IDEA se limita al diagnóstico bioquímico. Todas las instituciones a excepción del LGH-IVIC tienen áreas para consultas médicas especializadas relacionadas (CMR), para interconsultas (cuadro 2) de pediatría y ginecoobstetricia en el caso del IIG-LUZ, estudios por imágenes y neuropediatría en el CNGMV, mientras que los médicos de la UDEIM-IDEA se orientan al área de los errores congénitos del metabolismo. El CNGMV posee consultas profesionales relacionadas (CPR) como psicología y terapia ocupacional. No se pudo conocer el número de pacientes atendidos en estos servicios. Los profesionales de laboratorio de todas las instituciones se han formados en licenciaturas de biología, bioquímica, bioanálisis, enfermería y antropología.

En el caso de los servicios de diagnóstico, la totalidad de las instituciones de investigación cuenta con diagnóstico molecular (SDM) y de errores congénitos del metabolismo (ECM). El IIG-LUZ es la

CUADRO 1. Recurso humano de genética médica en el sector público según la profesión y el nivel de formación, 2016

\begin{tabular}{|c|c|c|c|c|c|c|c|c|c|c|c|c|c|}
\hline \multirow{2}{*}{ Instituciones } & \multirow{2}{*}{ Estado } & \multicolumn{5}{|c|}{ Médicos } & \multirow{2}{*}{ Subtotal } & \multicolumn{5}{|c|}{ Profesionales de laboratorio } & \multirow{2}{*}{ Subtotal } \\
\hline & & Ent & Esp & $\mathrm{MSc}$ & $\mathrm{PhD}$ & PD & & Tec & Lic & MSc & PhD & PD & \\
\hline \multicolumn{14}{|l|}{ Instituciones de investigación } \\
\hline Laboratorio de Genética Humana del IVIC (LGH-IVIC) & Miranda & 2 & 1 & 0 & 1 & 0 & 4 & 2 & 1 & 3 & 1 & 5 & 12 \\
\hline Instituto de Investigaciones Genéticas de LUZ (IIG-LUZ) & Zulia & 0 & 0 & 10 & 0 & 1 & 11 & 2 & 3 & 9 & 1 & & 15 \\
\hline Unidad de Errores Innatos del Metabolismo del IDEA (UDEM-IDEA) & Miranda & 0 & 2 & 0 & 0 & 0 & 2 & 2 & 6 & 1 & 1 & & 10 \\
\hline Centro Nacional de Genética Médica de Venezuela (CNGMV) & Miranda & 0 & 1 & 19 & 0 & 0 & 20 & 2 & 1 & 0 & 0 & & 3 \\
\hline SUBTOTAL & & 2 & 4 & 29 & 1 & 1 & 37 & 8 & 11 & 13 & 3 & 5 & 40 \\
\hline \multicolumn{14}{|l|}{ Universidades } \\
\hline Departamento de Biología Celular (USB) & Dto. Capital & 0 & 0 & 0 & 0 & 0 & 0 & 3 & 6 & 1 & 12 & 3 & 25 \\
\hline Centro Nacional de Genética Humana y Experimental (UCV) & Dto. Capital & 0 & 0 & 2 & 0 & 0 & 2 & 0 & 0 & 0 & 0 & 0 & 0 \\
\hline Unidad de Genética Médica (ULA) & Mérida & 0 & 0 & 2 & 0 & 0 & 2 & 0 & 0 & 1 & 0 & 0 & 1 \\
\hline Unidad de Genética Médica (UCLA) & Lara & 2 & 0 & 1 & 1 & 0 & 4 & 0 & 0 & 0 & 0 & 0 & 0 \\
\hline Unidad de Genética Médica y Citogenética (UC) & Carabobo & 0 & 0 & 1 & 0 & 0 & 1 & 0 & 0 & 0 & 0 & 0 & 0 \\
\hline Laboratorio de Genética Humana/Unidad Proyecto Aragua (UC) & Aragua & 0 & 0 & 1 & 0 & 0 & 1 & 0 & 1 & 0 & 1 & 0 & 2 \\
\hline Unidad de Genética Médica/Núcleo Bolívar (UDO) & Bolívar & 3 & 1 & 0 & 0 & 0 & 4 & 0 & 0 & 0 & 0 & 0 & 0 \\
\hline SUBTOTAL & & 5 & 1 & 7 & 1 & 0 & 14 & 3 & 7 & 2 & 13 & 3 & 28 \\
\hline \multicolumn{14}{|l|}{ Hospitales } \\
\hline Unidad de Genética/Hospital Dr. José María Vargas & Dto. Capital & 1 & 0 & 0 & 1 & 0 & 2 & 0 & 0 & 0 & 0 & 0 & 0 \\
\hline Hospital Clínico Universitario de Caracas (HCUC) & Dto. Capital & 0 & 1 & 0 & 0 & 0 & 1 & 0 & 0 & 0 & 0 & 0 & 0 \\
\hline Hospital Universitario Dr. Pedro Emilio Carrillo & Trujillo & 0 & 0 & 1 & 0 & 0 & 1 & 0 & 0 & 0 & 0 & 0 & 0 \\
\hline Servicio de Gineco-Obstetricia/Hospital Dr. Rafael Calles Sierra & Falcón & 0 & 0 & 1 & 0 & 0 & 1 & 0 & 0 & 0 & 0 & 0 & 0 \\
\hline SUBTOTAL & & 1 & 1 & 2 & 1 & 0 & 5 & 0 & 0 & 0 & 0 & 0 & 0 \\
\hline TOTAL & & 8 & 6 & 38 & 3 & 1 & 56 & 11 & 18 & 15 & 16 & 8 & 68 \\
\hline
\end{tabular}

Cuadro elaborado por los autores a partir de los resultados presentados.

Ent, entrenamiento; Esp, especialista; MSc, maestría; PhD, doctorado; PD, posdoctorado; Tec, técnico; Lic, licenciado; IVIC, Instituto Venezolano de Investigaciones Científicas; LUZ,

La Universidad del Zulia; IDEA, Fundación Instituo de Estudios Avanzados. 
CUADRO 2. Instituciones de investigación en genética médica según los servicios de atención y diagnóstico, 2016

\begin{tabular}{|c|c|c|c|c|c|c|c|c|c|}
\hline \multirow{2}{*}{ Instituciones } & \multicolumn{3}{|c|}{ Servicios de atención } & \multicolumn{6}{|c|}{ Servicios de diagnóstico } \\
\hline & CGM & CMR & CPR & SDC & SDM & SGF & DPN & DPNNI & ECM \\
\hline Laboratorio de Genética Humana del IVIC (LHG-IVIC) & $\cdot$ & & & & $\cdot$ & & & & - \\
\hline Instituto de Investigaciones Genéticas de LUZ (IIG-LUZ) & • & • & & • & • & • & • & • & • \\
\hline Unidad de Errores Innatos del Metabolismo del IDEA (UDEIM-IDEA) & & • & & & • & & & & - \\
\hline Centro Nacional de Genética Médica de Venezuela (CNGMV) & • & - & • & • & • & & & • & • \\
\hline
\end{tabular}

Cuadro elaborado por los autores a partir de los resultados presentados.

IVIC, Instituto Venezolano de Investigaciones Científicas; LUZ, La Universidad del Zulia; IDEA, Fundación Instituto de Estudios Avanzados; CGM, consulta de genética médica; CMR, consulta médica especializadas relacionadas; CPR, consultas profesionales relacionadas; SDC, servicio de diagnóstico citogenético; SDM, servicio de diagnóstico molecular; SGF, servicio de Genética Forense; DPN, servicio de diagnóstico prenatal; DPNNI, servicio de diagnóstico prenatal no invasivo; ECM, errores congénitos del metabolismo.

única institución que posee los servicios de diagnóstico prenatal (DPN) invasivo y no invasivo (DPNNI); el CNGMV brinda servicios de DPNNI mediante ecografía de primer y segundo trimestre de gestación, a su vez estas dos instituciones son las únicas que ofrecen el servicio de diagnóstico citogenético (SDC) convencional. El IIG-LUZ tiene servicios de genética forense (SGF) para determinar paternidad (cuadro 2).

El servicio de diagnóstico molecular es el más disponible, cada institución usa técnicas convencionales de biología molecular; basadas en la reacción en cadena de la polimerasa (PCR), con diferentes métodos diagnósticos de secuencias específicas de $\mathrm{ADN}$, como digestión con enzimas de restricción, amplificación, separación electroforética y transferencia a membranas. El LGH-IVIC está en capacidad de diagnosticar 35 condiciones genéticas, el IIG-LUZ puede identificar 11, mientras que el CNGMV y la UDEIM-IDEA lo hacen en cinco y dos condiciones, respectivamente (cuadro 3). El servicio de diagnóstico de errores congénitos del metabolismo es el segundo más disponible. El LGH-IVIC tiene una amplia capacidad de diagnóstico y puede hacerlo para 23 enfermedades metabólicas. El MPPS promueve la realización de cinco pruebas: para hipotiroidismo

congénito, fenilcetonuria, galactosemia, hiperplasia suprarrenal congénita y déficit de biotinidasa. En ese sentido, la UDEIM-IDEA es el centro de referencia del programa de tamizaje nacional de errores congénitos del metabolismo con el diagnóstico neonatal de fenilcetonuria e hipotiroidismo congénito, mediante método fluorométrico. Las muestra se toman de sangre periférica en las primeras 72 horas de vida del recién nacido, además realizan pesquisas selectivas para aminoacidemias, acidemias orgánicas, homocistinuria, deficiencia de biotinidasa y alteraciones de lactato/piruvato; el IIG-LUZ tiene a disposición técnicas bioquímicas para detectar alteraciones del metabolismo de carbohidratos, aminoácidos y ácidos orgánicos, así como de hemoglobinopatías, enfermedades de depósito como todas las mucopolisacaridosis y algunas lipofuscinosis; las técnicas del CNGMV son semicuantitativas y requieren de posteriores estudios para hacer diagnóstico (cuadro 3).

La distribución de recursos humanos a nivel nacional — sectores público y privado- según población y regiones del país, indicó que la región Zuliana presenta el mayor número de médicos genetistas a razón de millón de habitantes $(4,05)$, seguido por la región Capital $(3,42)$. En el caso de los genetistas de laboratorio los

CUADRO 3. Instituciones de investigación en genética médica según el número de técnicas diagnósticas de los servicios más disponibles, 2016

\begin{tabular}{lcc}
\hline \multirow{2}{*}{ Instituciones } & \multicolumn{2}{c}{ Servicios diagnósticos } \\
\cline { 2 - 3 } & SDM & ECM \\
\hline Laboratorio de Genética Humana del IVIC (LGH-IVIC) & 35 & 23 \\
Instituto de Investigaciones Genéticas de LUZ (IIG-LUZ) & 11 & 6 \\
Unidad de Errores Innatos del Metabolismo del IDEA (UDEIM-IDEA) & 2 & 8 \\
Centro Nacional de Genética Médica de Venezuela (CNGMV) & 5 & 2 \\
\hline
\end{tabular}

Cuadro elaborado por los autores a partir de los resultados presentados.

IVIC, Instituto Venezolano de Investigacines Científicas; LUZ, La Universidad del Zulia; IDEA, Fundación Instituto de

Estudios Avanzados; SDM, servicio de diagnóstico molecular; ECM, errores congénitos del metabolismo. valores se invierten, con la región Capital con mayor cantidad a razón de millón de habitantes $(10,06)$, seguido de la Zuliana $(4,05)$. Las regiones Insular y de los Llanos no cuentan con profesionales formados en genética y las regiones Centro-Occidental, de Guayana y NorOriental no disponen de genetistas de laboratorio (cuadro 4). Las regiones $\mathrm{Zu}$ liana, Capital, de los Andes, Centro-Occidental y de Guayana tienen entre dos y cuatro médicos genetistas por millón de habitantes.

Las instituciones venezolanas que ofrecen formación en genética son el LGH-IVIC y el IIG-LUZ. El LGH-IVIC desde 1974 tiene un programa de formación de maestros (MSc) y doctores (PhD por sus siglas en inglés) con mención genética humana, en los cuales han egresado 36 MSc y 10 PhD hasta 2016. Así mismo, el IIG-LUZ en 1992 creó una maestría dirigida a médicos con mención genética médica, donde se han formado 26 MSc; en 2001 inicio una segunda maestría con mención genética humana dirigida a profesionales de laboratorio, capacitando 19 MSc en 15 años.

\section{DISCUSIÓN}

En América Latina y el Caribe los servicios de genética médica tienden a concentrarse en grandes ciudades, tener alcance limitado y el sector público ser el mayor proveedor de servicios (3). Venezuela coincide con este comportamiento, siendo que tres de las cuatro instituciones de investigación se localizan en el estado Miranda en la región Capital (figura 1), la de mayor densidad poblacional (cuadro 4). Tienen en común el difícil acceso por ubicarse en la periferia de la ciudad, posiblemente causado por una deficiente planificación y escasas políticas públicas dirigidas en este sentido. La región Capital tiene una población aproximada de 4,9 
CUADRO 4. Distribución de recursos humanos en genética médica según la población y la región del país, 2016

\begin{tabular}{|c|c|c|c|c|c|c|c|c|}
\hline \multirow[t]{2}{*}{ Región } & \multirow[t]{2}{*}{ Estados y áreas de conformación } & \multirow{2}{*}{$\begin{array}{l}\text { Superficie } \\
\left(\mathrm{km}^{2}\right)\end{array}$} & \multirow[t]{2}{*}{ Población } & \multirow{2}{*}{$\begin{array}{r}\text { Densidad } \\
\left(\mathrm{hab} / \mathrm{km}^{2}\right)\end{array}$} & \multicolumn{2}{|c|}{ Médicos } & \multicolumn{2}{|c|}{$\begin{array}{l}\text { Profesionales de } \\
\text { laboratorio }\end{array}$} \\
\hline & & & & & $N$ & $\mathrm{pmh}$ & $N$ & $\mathrm{pmh}$ \\
\hline Zuliana & Zulia & $50230^{a}$ & 3704404 & 73.7 & 15 & 4.05 & 15 & 4.05 \\
\hline de los Andes & Barinas, Mérida, Táchira, Trujillo y el municipio Páez de Apure & 77820 & 3600256 & 46.3 & 8 & 2.22 & 1 & 0.28 \\
\hline Centro-Occidental & Falcón, Lara, Portuguesa y Yaracuy & 66900 & 4155062 & 62.1 & 11 & 2.65 & 0 & 0.00 \\
\hline Central & Aragua, Carabobo y Cojedes & $26089^{b}$ & 4199217 & 161.0 & 6 & 1.43 & 2 & 0.48 \\
\hline Capital & Distrito Capital, Miranda y Vargas & 9880 & 4971986 & 503.2 & 17 & 3.42 & 50 & 10.06 \\
\hline de los Llanos & Apure y Guárico & 128666 & 1106639 & 8.6 & 0 & 0.00 & 0 & 0.00 \\
\hline de Guayana & Amazonas, Bolívas y Delta Amacuro & 458345 & 1725120 & 3.8 & 4 & 2.32 & 0 & 0.00 \\
\hline Nor-Oriental & Anzoátegui, Monagas y Sucre & 84000 & 3271481 & 38.9 & 2 & 0.61 & 0 & 0.00 \\
\hline Insular & Nueva Esparta y las dependencias federales & 1270 & 493765 & 388.8 & 0 & 0.00 & 0 & 0.00 \\
\hline
\end{tabular}

Cuadro elaborado por los autores a partir de datos publicados.

Fuentes: Centro Nacional de Tecnologías de Información e Instituto Nacional de Estadística.

km², kilómetros cuadros; hab/km², habitantes por kilómetro cuadrado; pmh, por millón de habitantes.

a No incluye $12870 \mathrm{~km}^{2}$ correspondientes a la supeficie del lago de Maracaibo.

${ }^{\mathrm{b}}$ No incluye $375 \mathrm{~km}^{2}$ correspondientes a la superficie del lago de Valencia.

FIGURA 1. Mapa de la República Bolivariana de Venezuela, regiones político-administrativas, 2016

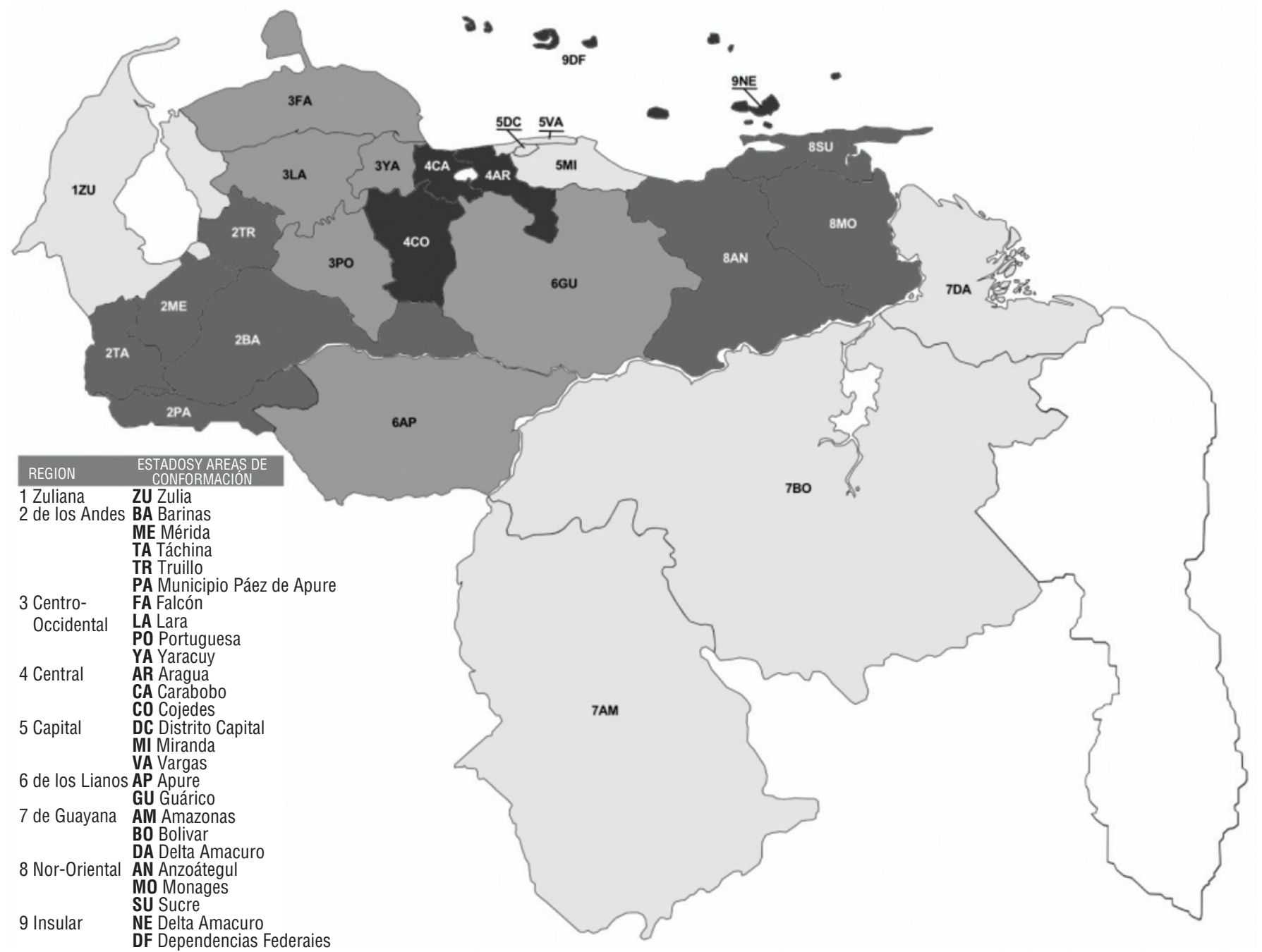

Fuentes: Centro Nacional de Tecnologías de Información.

Elaborada por los autores a partir de datos publicados. 
millones de habitantes, que representan 1,6 millones de habitantes por cada centro de genética, esto supera la meta recomendada de centros por millón de habitantes (5). Más de la mitad de los recursos humanos $(62 \%)$ trabajan en estas instituciones.

En Venezuela, cinco regiones superan el estimado para América Latina de médicos genetistas por millón de habitantes (8) y el $10 \%$ ejercen tanto en el sector público como en el privado. El 96\% de los genetistas de laboratorio se distribuyen en dos regiones. Por otra parte, las regiones Insular y de los Llanos no tienen profesionales formados en genética, lo que significa que hay 1,5 millones de habitantes sin acceso a estos servicios. El CNGMV se apoya en profesionales cubanos y cuenta con cuatro médicos genetistas e igual número de genetistas de laboratorio; su cooperación transitoria es fluctuante y rotativa, por lo cual no han sido incluidos en el reporte. Conocer la demanda de los servicios fue una limitación de la investigación, en parte porque algunos centros no pudieron facilitar la información.

El 40\% de los profesionales de genética que laboran en la actualidad se han formado tanto en el IIG-LUZ como en el LGH-IVIC, evidencia de su contribución en el crecimiento del área en las últimas cuatro décadas. La mitad de los recursos humanos formados en la maestría del IIG-LUZ se han incorporado a la institución como investigadores y docentes y conforman un claustro de 18 profesores, de esta manera se garantiza la transmisión de conocimientos a las nuevas generaciones. El CNGMV ha formado el recurso humano en el Centro Nacional de Genética Médica de La Habana mediante un programa dirigido a médicos (18); se han graduado cuatro especialistas en genética clínica, 29 MSc en asesoramiento genético y seis médicos entrenados en citogenética. La formación de recursos y la colaboración transnacional en investigación biomédica son factores trascendentales en el desarrollo de sistemas de atención en genética médica $(19,20)$. La comisión de expertos de la Red Latinoamericana de Genética Humana (RELAGH) y la Organización Mundial de la Salud (OMS) promueven en la región la creación de programas de entrenamiento en genética médica dirigido a diversos profesionales de la salud (21).

La institución con el mayor número de profesionales que han migrado es el CNGMV, 19 en total; de estos, tres especialistas en genética clínica, diez MSc en asesoramiento genético y la totalidad de médicos entrenados en citogenética. Dos egresados de la maestría dictada por el IIG-LUZ ha emigrado del país. En la fundación IDEA, diez profesionales con nivel de PhD han dejado la institución, lo que afectó la operatividad de un laboratorio de genética forense inaugurado en 2013. La migración profesional fuera del territorio ha sido considerable en los últimos cinco años, equivalente al 25\% de los recursos actualmente disponibles en el sector público.

El CNGMV dirige un programa de genética de base poblacional en prevención primaria mediante una red de genética comunitaria, que busca reducir la brecha de acceso a los servicios. Desde 2009, la red fue atendida por médicos cubanos con nivel de MSc en asesoramiento genético, organizados a razón de un médico por estado, para un total de 24 (en carácter transitorio); en 2011 se incorporaron 19 médicos venezolanos con MSc en asesoramiento genético en diez estados del país. La organización de esta red trata de emular el funcionamiento de los servicios de genética médica en Cuba (22). En la actualidad, el MSc en asesoramiento genético no tiene reconocimiento oficial en Venezuela.

La UDEIM-IDEA desde 1985 forma parte del programa de genética de base poblacional de errores congénitos del metabolismo. Hoy en día, es centro referencia nacional para pesquisa neonatal y confirma muestras a nivel nacional de los laboratorios de salud pública y hospitales adscritos al MPPS. El IIGLUZ participa en este programa y ha estudiado a más de 30000 neonatos hasta enero de 2016, este tamizaje forma parte de los programas de genética de prevención secundaria, similar a las experiencias llevadas por países de la Región (23). Por otra parte, El IIG-LUZ se ha abocado al estudio clínico epidemiológico de la enfermedad de Huntington, enmarcado en los programas de genética de prevención terciaria; cuenta incluso con la prueba presintomática de la enfermedad, atendiendo las comunidades de la costa occidental del lago de Maracaibo, donde se reporta la prevalencia más alta de la enfermedad en el mundo (24). El SPNS carece de indicadores de incidencia o prevalencia de otras enfermedades genéticas y no dispone de una legislación en materia de pesquisa neonatal, anomalías congénitas o enfermedades genéticas. Desde 2008, está vigente un código de bioética y bioseguridad (25) que regula de manera general la investigación en seres humanos, sin hacer referencia a la práctica de la genética médica.

En 2016 la esperanza de vida al nacer en Venezuela era de 74,6 años (mujeres: 78,8; hombres: 70,6). En el último reporte de mortalidad por 1000 nacidos vivos, la tasa de mortalidad infantil se ubicó en 14,7; la tasa de mortalidad neonatal en 10,7 y la mortalidad en menores de cinco años fue de 16,9 (26). En menores de 28 días de vida, las principales causas de mortalidad fueron afecciones perinatales $(79,93 \%)$ y anomalías congénitas $(17,52 \%)$; se mantuvieron ambas en menores de un año con valores de $60,30 \%$ y 20,40\% respectivamente; de uno a cuatro años, los accidentes de todo tipo $(20,27 \%)$, las anomalías congénitas $(15,51 \%)$ e influenza y neumonía $(12,35 \%)$ son las principales causas de mortalidad infantil. En 2011, se registró una tasa de muertes por anomalías congénitas de 10,5 por cada 100000 nacidos vivos $(16,27)$, en 2013 se reportaron 2428 muertes por anomalías congénitas (28). No existe una política nacional de vigilancia epidemiológica de anomalías congénitas, siendo estas de las principales causas de muerte en menores de cinco años. Venezuela es parte del Estudio Colaborativo Latinoamericano de Malformaciones Congénitas (ECLAMC) desde 1973 (29), en 2015 tan solo un hospital reportaba al ECLAMC (30). La interrupción voluntaria del embarazo es ilegal en Venezuela y está permitida solo en situaciones de riesgo para la vida de la gestante.

En Venezuela existe un amplio grupo de profesionales formados en genética médica, pero su número se ha visto reducido en los últimos cinco años. Estos recursos se localizan en su mayoría en las instituciones de investigación, con considerable tecnología y acceso limitado. La contribución de las instituciones de investigación a la formación de profesionales en genética es significativa. Sería recomendable plantearse un modelo organizativo que incluya al MPPS e integre las instituciones de investigación y centros de genética e incorporarlos al nivel de mayor complejidad del SPNS, con el fin de reducir la brecha de acceso a los servicios. Esto permitiría incrementar la creación de grupos de investigación multidisciplinarios, la interacción entre comités de ética de la investigación, la creación y/o unificación de registros de defectos congénitos y programas preventivos de base poblacional, así como la coordinación de formación de recursos humanos. 
Agradecimientos. A Víctor Penchaszadeh por sus valiosos aportes y sugerencias. A la cátedra de Gestión en Salud Pública del Servicio Autónomo Instituto de Altos Estudios “Dr. Arnoldo Gabaldón". A la Sociedad Venezolana de
Genética Humana y las instituciones de investigación que colaboraron con la investigación.

Conflicto de intereses. Ninguno declarado por los autores.

\section{REFERENCIAS}

1. Griffiths AJF, Wessler SR, Lewontin RC, Carroll SB. Genética. 9a ed. Madrid: McGraw Hill; 2008.

2. McKusick V, Harper P. History of medical genetics. En: Rimoin D, Pyeritz R, Korf B, editores. Emery and Rimoin's Principles and Practice of Medical Genetics. 6th ed. Vol. 1. San Diego: Academic Press; 2013:1.

3. Penchaszadeh VB. Genetic services in Latin America. J Community Genet. 2004;7(2-3):65-9. doi: 10.1159/000080773

4. Epstein C. Medical genetics in the genomic medicine of the 21st century. Am J Hum Genet. 2006;79(3):434-8. doi: 10.1086/507610

5. Penchaszadeh VB. Servicios de genética médica. En: O'Donnell JO, editores. Administración de Servicios de Salud. Tomo III. Buenos Aires: Fundación Garrahan; 1999:194-210.

6. Ejarquea I, García-Ribesa M, Martín V. El médico de familia y sus competencias en genética clínica. Aten Primaria. 2007;39(3):113-4.

7. Penchaszadeh VB. Community genetics in Latin America: challenges and perspectives. Community Genet. 2000;3:124-7.

8. Penchaszadeh VB. Ethical issues in genetics and public health in Latin America with a focus on Argentina. J Community Genet. 2015;6(3):223-30. doi: 10.1007/ s12687-015-0217-5

9. Organización Panamericana de la Salud (OPS). Salud en las Américas, 2017. Resumen: panorama regional y perfiles de país. Washington, D.C.: OPS; 2017. Disponible en: http://www.paho.org/salud-en-las-americas-2017/?page_id=4235\&lang=es Acceso el 28 de diciembre de 2017.

10. Ministerio del Poder Popular para Educación Universitaria, Ciencia y Tecnología de Venezuela (MPPEUCT) e Instituto Venezolano de Investigaciones Científicas. Laboratorio de Genética Humana Histórica. Caracas: MPPEUCT; 2014. Disponible en: http://www.ivic. gob.ve/medicina/genetica / ? mod=historia.htm Acceso el 6 de julio de 2014.

11. Ministerio del Poder Popular para Educación Universitaria, Ciencia y Tecnología de Venezuela (MPPEUCT) y Consejo Nacional de Universidades (CNU). Secretariado Permanente del CNU: Resolución 474. Caracas: MPPEUCT; 2012. Disponible en: http:/ / www.spcnu.gob.ve/ index.php/banco-de-documentos/resoluciones-cnu/2012/resolucion-n-474-mayo/ resolucion-n-474-mayo/download Acceso el 9 de marzo de 2017.

12. Ministerio del Poder Popular para Educación Universitaria, Ciencia y Tecnología de Venezuela (MPPEUCT) y Fundación Instituto de Estudios Avanzados. Historia. Caracas: MPPEUCT; 2016. Disponible en: http: / / www.idea.gob.ve/contenido. php?pag=5 Acceso el 8 de agosto de 2016.

13. Embajada de la República de Cuba en la República Bolivariana de Venezuela. Convenio Integral de Cooperación. Caracas: Embajada de la República de Cuba en la República Bolivariana de Venezuela; 2000. Disponible en: http://www.embajadacuba. com.ve/cuba-venezuela/convenio-colaboracion/ Acceso el 9 de marzo de 2017.

14. Falcón de Vargas A. Genética y Genómica en Latinoamérica Hoy. Conferencia presentada en el IX Curso de la Escuela Latinoamericana de Genética Humana y Médica; 2013 Mayo 5-11; Porto Alegre, Brasil. Porto Alegre: Instituto Genética Para Todos; 2013.

15. Penchaszadeh VB, Heredero L, PunalesMorejon D, Rojas I, Perez ET. Genetic counseling training in Cuba. Am J Hum Genet. 1997;61(4)SA:1099.

16. Ministerio del Poder Popular dePlanificación (MPPP) e Instituto Nacional de Estadística. XIV Censo Nacional de Población y Vivienda: Resultados Total Nacional 2011. Caracas: MPPP; 2014. Disponible en: http:/ / www.ine.gov.ve/documentos/Demografia /CensodePoblacionyVivienda/pdf/nacional.pdf Acceso el 20 de marzo de 2017.

17. Ministerio del Poder Popular para Educación Universitaria, Ciencia y Tecnología (MPPEUCT) y Centro Nacional de Tecnologías de Información. Regiones geográficas. Caracas: MPPEUCT; 2015. Disponible en: http://gobiernoenlinea. gob.ve/home/geografia2.dot Acceso el 15 de diciembre de 2016.

18. Lantigua-Cruz A. An overview of genetic counseling in Cuba. J Genet Couns. 2013; 22:849-53.

19. Sirisena ND, Dissanayake VHW. Focusing attention on ancestral diversity within genomics research: a potential means for promoting equity in the provision of genomics based healthcare services in developing countries. J Community Genet. 2017;8:27581. doi: 10.1007/s12687-017-0311

20. Mitropoulos K, Al Jaibeji H, Forero DA, Laissue P, Wonkam A, López-Correa C, et al. Success stories in genomic medicine from resource-limited countries. Hum Genomics. 2015;18(9):11. doi: 10.1186/ s40246-015-0033-3

21. Kofman-Alfaroa S, Penchaszadeh VB. Community genetic services in Latin America and regional network of medical genetics. Community Genet 2004;7:157159. doi: 10.1159/000080789

22. Lantigua-Cruz A, Lemus-Valdés MT, Marcheco-Teruel B. Medical genetic services in Cuba. Rev Cubana Genet Comunit. 2007;1(1):15-19.
Declaración. Las opiniones expresadas en este manuscrito son responsabilidad de los autores y no reflejan necesariamente los criterios ni la política de la RPSP/PAJPH o de la OPS.
23. Penchaszadeh VB, Beiguelman B. Medical genetic services in Latin America: report of a meeting of experts. Pan Am J Public Health. 1998;3(6):409-20.

24. Pineda-Bernal L. Análisis bioético de la investigación de la enfermedad de Huntington en el Estado Zulia, Venezuela. Rev Redbioética/UNESCO. 2010;1(2):50-61.

25. Ministerio del Poder Popular para Educación Universitaria, Ciencia y Tecnología (MPPEUCT). Código de Bioética y Bioseguridad. Caracas: MPPEUCT; 2008. Disponible en: http://www.ciens.ucv.ve: 8080 / generador/sites / biolanimlab / archivos/codigo_fonacit_2008.pdf Acceso el 28 de diciembre de 2017.

26. Organización Panamericana de la Salud/ Organización Mundial de la Salud. Enfermedades transmisibles y análisis de salud/Información y análisis de salud: situación de salud en las Américas, indicadores básicos 2017. Washington, D.C., Estados Unidos de América, 2017. Disponible en: http://iris.paho.org/ xmlui/bitstream/handle/123456789/ 34330 / IndBrasicos2017_spa.pdf? sequence $=1 \&$ is Allowed $=y$ Acceso el 28 de diciembre de 2017.

27. Ministerio del Poder Popular para la Salud de Venezuela (MPPS). Anuario de Mortalidad 2011. Caracas: MPPS; 2014. Disponible en: https:/ /www.ovsalud.org/ descargas/publicaciones/documentosoficiales / Anuario-Mortalidad-2011.pdf Acceso el 29 de diciembre de 2017.

28. Ministerio del Poder Popular para la Salud de Venezuela (MPPS). Anuario de Mortalidad 2013. Caracas: MPPS; 2015. Disponible en: https: / /www.ovsalud.org/ descargas/publicaciones/documentosoficiales / Anuario-Mortalidad-2013.pdf Acceso el 29 de diciembre de 2017.

29. Castilla EE, Orioli IM. ECLAMC: The Latin-American Collaborative Study of Congenital Malformations. Community Genet.2004;7:76-94.doi:10.1159/000080776

30. Poletta FA. Situación Actual del Estudio Colaborativo Latino Americano de Malformaciones Congénitas (ECLAMC). Conferencia presentada en la $47^{\mathrm{a}}$ Reunión Anual del ECLAMC; 2015 noviembre 5-6; Buenos Aires, Argentina. Buenos Aires: Instituto Universitario CEMIC; 2015.

Manuscrito recibido el 19 de setiembre de 2017. Aceptado para su publicación, tras revisión, el 12 de marzo de 2018 
ABSTRACT Objective. To characterize medical genetics services in Venezuela and describe the distribution of their resources, services, technologies, and professional training.

Methods. A descriptive, documentary study of genetic services was conducted

Medical genetics services in Venezuela

between February and November 2016, involving a review of primary documentary sources and the use of a data collection form in research institutions to obtain information on the availability of human resources, clinical care, and diagnostic services, as well as professional training. Furthermore, the Venezuelan Society of Human Genetics database was used to identify the human resources available in genetics centers. The criterion for inclusion was being an institution with staff trained in genetics.

Results. The inclusion criterion was met by four research institutions, seven universities, and four hospitals, all in the public sector. A total of 124 professionals work in these institutions; 56 of them are physicians and 68 are laboratory staff. Of these professionals, $62 \%$ are affiliated with research institutions, which offer patient care services, molecular and biochemical diagnostic services, and, more rarely, cytogenetic, prenatal, and forensic testing. Five regions of the country have between two and four physicians specializing in genetics per million inhabitants. Of the laboratory professionals, 96\% are located in two regions (Capital and Zuliana); five regions have none. Research institutions have provided training in genetics to $40 \%$ of the country's current human resources.

Conclusions. Genetics services show great variability in terms of diagnostic options. They train large numbers of professionals, but access is limited. There is a need for coordinated policies to integrate these services and reduce existing gaps.

Keywords Genetics, medical; genetic services; human resources; Venezuela.

RESUMO Objetivo. Caracterizar os serviços de genética médica da Venezuela com a finalidade de conhecer a distribuição dos recursos, prestação de serviços, tecnologias usadas e formação profissional nesta área.

\section{Serviços de genética médica na Venezuela}

\author{
Palavras-chave
}

Métodos. Uma pesquisa descritiva documental dos serviços de genética médica foi realizada de fevereiro a novembro de 2016. Foi feita uma revisão das fontes documentais primárias nas instituições de pesquisa com o preenchimento de fichas de coleta de dados com informação sobre a disponibilidade de recursos humanos, prestação de serviços de atendimento e diagnóstico e formação profissional. Foi feita também uma revisão do banco de dados da Sociedade Venezuelana de Genética Humana para identificar os recursos humanos nos centros de genética. O critério de inclusão do estudo foi ser uma instituição com recursos humanos formados em genética.

Resultados. Os critérios do estudo foram satisfeitos por 4 instituições de pesquisa, 7 universidades e 4 hospitais, todos da rede pública. Foram identificados 124 profissionais trabalhando nestas instituições: 56 médicos e 68 funcionários da área de laboratório. Sessenta e dois por cento dos profissionais pertencem a instituições de pesquisa que prestam serviços de atendimento clínico e diagnóstico molecular e bioquímico e, menos frequentemente, realizam análises citogenéticas, pré-natais e forenses. Cinco regiões do país têm entre 2 e 4 médicos geneticistas por milhão de habitantes. Além disso, 96\% dos profissionais de laboratório estão distribuídos em 2 regiões (capital e Zuliana), sendo que não há nenhum profissional em 5 regiões. As instituições de pesquisa formam atualmente $40 \%$ dos recursos humanos em genética do país. Conclusões. Os serviços de genética médica são caracterizados por grande variabilidade nas opções diagnósticas, acesso limitado e grande contribuição para a formação profissional. Fazem-se necessárias políticas coordenadas para integrar e reduzir as lacunas. 